\title{
Residência Médica na Área de Cancerologia no Brasil: Distribuição dos Programas e da Oferta de Vagas por Região em 2010
}

Medical Residency in Cancerology in Brazil: Distribution of the Programs and Current offer of Openings According to Region in 2010 Adhesión a la Terapia Residencia Medica en el Área de Cancerología en Brasil: Distribución de los Programas y la Oferta del Número de Plazas por Región en 2010

\author{
Marcelo Gurgel Carlos da Silva'; Miren Maite Uribe Arregi'i ${ }^{2}$ Carlos Márcio Melo de Matos ${ }^{3}$
}

\section{Resumo}

Introduçáo: A residência médica constitui modalidade de ensino de pós-graduação, destinada a médicos, sob a forma de cursos de especialização, caracterizada por treinamento em serviço, funcionando sob a responsabilidade de instituiçóes de saúde, universitárias ou não, sob a orientação de profissionais médicos de elevada qualificação profissional. Objetivos: Analisar a oferta de programas e vagas de residência médica em cancerologia, no Brasil, em 2010, comparando com a estimativa de população, incidência e óbitos por câncer, segundo macrorregióes; além de comparar a distribuição desses programas, segundo dependência administrativa e de avaliar a distribuição dos Centros de Alta Complexidade em Oncologia. Método: Trata-se de um estudo descritivo e quantitativo do tipo transversal. Os dados obtidos foram dispostos em planilhas e correlacionados através de análises estatísticas. Quando indicado, foi feito o teste estatístico pela correlação de Spearman. Resultados: A região Sudeste congrega 59,8\% dos programas; a regiāo Sul abriga 16,3\%; o Centro-Oeste, $7,18 \%$; o Nordeste, $14,8 \%$; e a região Norte, $1,91 \%$. Considerando-se as áreas de cancerologia, cancerologia clínica, cancerologia pediátrica, cancerologia cirúrgica e radioterapia; viu-se que, entre 2003 e 2010, houve um incremento de vagas de 90,86\%. Conclusão: A região Sudeste detém a maioria dos programas e vagas e dos Centros de Alta Complexidade em Oncologia. É a única do País que possui um maior valor percentual de programas e vagas em relação ao valor percentual que detém da população brasileira, do total de óbitos por câncer e da incidência de câncer.

Palavras-chave: Oncologia-Educação; Internato e Residência; Prática Profissional; Capacitação em Serviço

\footnotetext{
${ }^{1}$ Doutor em Saúde Pública. Diretor de Ensino e Pesquisa do Instituto do Câncer do Ceará. Fortaleza (CE), Brasil. E-mail: marcelo@hospcancer-icc. org.br.

2 Mestre em Saúde Pública. Médica Epidemiologista do Instituto do Câncer do Ceará. Fortaleza (CE), Brasil. E-mail: mirenuribe@yahoo.com.br.

3 Acadêmico de Medicina. Universidade Estadual do Ceará. Fortaleza (CE). Brasil. E-mail: carlossmarcio@gmail.com.

Trabalho realizado no Instituto do Câncer do Ceará. Fortaleza (CE), Brasil.

Endereço para correspondência: Instituto do Câncer do Ceará. Rua Papi Júnior, 900 - Rodolfo Teófilo. Fortaleza (CE), Brasil. CEP: 60430-230.
} 


\section{INTRODUÇÃO}

A residência médica constitui modalidade de ensino de pós-graduação, destinada a médicos, sob a forma de cursos de especialização, caracterizada por treinamento em serviço, funcionando sob a responsabilidade de instituiçóes de saúde, universitárias ou não, sob a orientação de profissionais médicos de elevada qualificação ética e profissional ${ }^{1}$.

A residência médica originou-se nos Estados Unidos, no século XIX, como um sistema de instrução baseado no ensino clínico e no aproveitamento da rede hospitalar para o ensino médico ${ }^{2-3}$. No Brasil, as primeiras experiências de residência médica datam da década de 1940 . O Hospital das Clínicas da Universidade de São Paulo e o Hospital dos Servidores do Estado, no Rio de Janeiro, foram os primeiros a implantar o programa de residência médica, em 1944 e 1948, respectivamente ${ }^{2-3}$. Na área da cancerologia, os primeiros programas foram os do Hospital A.C. Camargo, em São Paulo, em 1954 . Gradativamente, foi aumentando o número de programas de residência médica, até que na década de 1970 ocorreu um grande avanço, culminando com a criação da Comissão Nacional de Residência Médica (CNRM), em 1977, cuja finalidade seria disciplinar essa forma de capacitação².

Os serviços vinculados ao Sistema Único de Saúde (SUS), que realizam tratamento oncológico no Brasil, são cadastrados pelo Ministério da Saúde como Centros de Alta Complexidade em Oncologia (CACON), Serviços Isolados de Quimioterapia ou de Radioterapia, compondo uma Rede de Atendimento em Oncologia, importante tanto para o tratamento de doenças como para a formação de oncologistas.

Em 2005, foi publicado um artigo intitulado "Residência médica na área de oncologia no Brasil: distribuição dos programas e da oferta de vagas por região em 2003" ${ }^{5}$. Nesse trabalho, demonstrou-se a existência, no País, de 38 instituiçóes que congregavam 48 programas de residência na área de cancerologia (excluindo-se as áreas de cirurgia de cabeça e pescoço, mastologia e anatomia patológica) e contavam com 361 vagas. Viu-se que a distribuição desses era bastante desequilibrada, com uma grande concentração nas regiốes Sul-Sudeste.

Este trabalho tem como objetivos: analisar a oferta de programas e vagas de residência médica em cancerologia, no Brasil, em 2010, comparando com a estimativa de população, incidência e óbitos por câncer, segundo macrorregiôes; além de comparar a distribuição desses programas, segundo dependência administrativa (federal, estadual, municipal, filantrópico-privada e privada-sentido estrito) e de avaliar a distribuição dos CACON, por região, no Brasil.

\section{MÉTODO}

Trata-se de um estudo descritivo e quantitativo do tipo transversal. Os dados sobre os programas de residência médica no Brasil foram obtidos por acesso eletrônico ao site do Ministério da Educação ${ }^{6}$ que abriga a página da CNRM, espelhando a situação dos programas cadastrados em setembro de 2010. Para cada instituição, foram colhidos informes sobre dependência administrativa, localização, oferta de vagas e programas, na área de cancerologia. Foram retiradas do aludido sítio eletrônico, as várias denominaçóes em uso na área de cancerologia (cancerologia - nomenclatura atual, oncologia - antiga nomenclatura, cancerologia clínica, cancerologia cirúrgica, cancerologia pediátrica), além da radioterapia, cirurgia de cabeça e pescoço, mastologia e patologia, face à estreita vinculação desses com a cancerologia.

Os dados foram dispostos e ordenados em planilhas eletrônicas do Microsoft Excel. Os programas recentemente descredenciados pela CNRM e aqueles em diligência, para fins de credenciamento, nessa comissão, foram excluídos da análise dos resultados.

Efetuaram-se comparações com outros parâmetros regionais, como capacidade instalada em $\mathrm{CACON}^{7}$, população ${ }^{8}$, óbitos ${ }^{9}$ e casos esperados de câncer $^{10}$. Além disso, foi estabelecido um paralelo com os dados encontrados no trabalho: "Residência médica na área de oncologia no Brasil: distribuição dos programas e da oferta de vagas por região em 2003 "' .

Quando indicado, foi feito o teste estatístico pela correlação de Spearman, que melhor se ajusta à análise por postos. Para isso, os resultados da incidência de câncer por macrorregiāo, segundo sexo, foram recalculados, para a população total. Pela natureza do trabalho, envolvendo apenas banco de dados de domínio público, a submissão ao Comitê de Ética em Pesquisa é dispensada.

\section{RESULTADOS}

Ao todo, foram encontrados 209 programas de residência médica, em áreas da cancerologia. A região Sudeste congrega 59,8\% (125) dos programas de residência em alguma área ligada à oncologia; a região Sul abriga 34 (16,3\%); o Centro-Oeste, 15 (7,18\%); o Nordeste, 31 (14,8\%); e a região Norte, 4 (1,91\%).

O Estado com maior representação é São Paulo, com 82 programas (39,2\%); seguem- se a ele Minas Gerais, com 21 (10,04\%), e o Rio de Janeiro, com 19 (9,1\%). Cabe destacar que essa distribuição não reflete a oferta das vagas de residência médica.

Analisando a capacidade instalada, em termos de centros de alta complexidade em oncologia (CACON), observa-se que, do total de CACON, em cada regiáo, somente uma pequena parte oferece programa de residência médica em oncologia. No total, $22,4 \%$ das instituiçóes, com essa qualificaçấo, oferecem algum programa de residência. Assim, no Centro-Oeste, são 28,6\%; na região Norte, 16,7\%; no Nordeste, 10\%; no Sul, 24,4\%; e no Sudeste, 
$27,3 \%$. As regiôes Norte e Nordeste são as únicas que ficam abaixo dos $20 \%$ da média nacional.

A distribuição dos programas, segundo dependência administrativa, mostra, em primeiro lugar, as instituiçóes federais, com $41 \%$ dos programas. As instituiçóes estaduais vêm a seguir, representando 34\%. Logo em seguida, aparecem as instituiçôes filantrópico-privadas com $18 \%$ de representatividade. As instituiçóes privadas - sentido estrito - são responsáveis por $6 \%$ dos programas. Em último lugar, as instituições municipais assumem 1\% dos programas.

A distribuição de vagas anuais por Estado e por Região mostra um desequilíbrio ainda maior. Na regiâo Norte, apenas 17 vagas foram ofertadas; no Centro-Oeste, com 15 programas, o número de vagas chega 61 (5,04\%); o Nordeste conta com 127 vagas (10,5\%); a região Sul disponibiliza 158 vagas (13,05\%), enquanto a região Sudeste oferece mais de dois terços das vagas, 847 (70\%) (Tabela 1).

No que diz respeito ao número de vagas ofertado e à população, a região Norte detém $8,02 \%$ da população nacional, mas apenas $1,4 \%$ das vagas de residência médica em cancerologia. No Nordeste, a desigualdade é ainda maior, já que possui $27,9 \%$ da população do País e somente $10,32 \%$ das vagas. No Centro-Oeste, encontram-se 7,25\% dos brasileiros e 4,7\% das vagas ofertadas. Já no Sudeste, a situação se inverte, pois é ocupada por $42,26 \%$ da população do Brasil e oferta 69,94\% das vagas de residência médica em cancerologia. No Sul, é onde há a menor discrepância, pois possui $14,48 \%$ da população brasileira e oferece $14,53 \%$ das vagas (Tabela 2 ).

Quando comparados esses dados, com o número estimado de casos novos, em câncer, o desequilíbrio é menor (Figura 1 e Tabela 2). No entanto, é, de novo, a regiáo Sudeste a que detém um maior percentual de vagas ofertadas $(69,94 \%)$ em relação ao percentual de casos novos (50,68\%). A região Norte, em 2010, detinha 3,9\% dos casos novos de câncer e 1,4\% das vagas. O Nordeste teve $18,2 \%$ dos casos novos e oferece $10,32 \%$ das vagas. $O$ Centro-Oeste possui 6,2\% dos casos novos e oferta 4,7\%. A região Sul detém 20,9\% dos casos novos e 13,62\% das vagas (Tabela 2). Essa desigualdade foi confirmada através do teste de correlação de Spearman. Com efeito, usando, no referido teste, os seguintes valores de incidência por 100.000 habitantes: Norte $(117,73)$, Nordeste $(165,75)$, Centro-Oeste $(213,10)$, Sudeste $(295,94)$ e Sul $(357,53)$; obteve-se o resultado de 0,3 , o que indica a inexistência de correlação entre a incidência de câncer e a oferta de vagas em residência médica, por regiáo brasileira.

Em relaçáo ao número esperado de óbitos por câncer, a região Sudeste mostrou um maior percentual de vagas ofertadas $(69,94 \%)$ em relação ao percentual de mortes por neoplasia maligna $(48,99 \%)$ na região. As demais regióes têm um percentual maior de mortes por câncer em relação ao número de vagas ofertadas (Tabela 2 e Figura 2).

Tabela 1. Distribuição de programas e vagas (todas as áreas), segundo Estado (UF) e região, no Brasil, em 2010

\begin{tabular}{|c|c|c|c|c|c|}
\hline \multirow{2}{*}{ Região } & \multirow{2}{*}{ UF } & \multicolumn{2}{|c|}{ Programas } & \multicolumn{2}{|c|}{ Vagas } \\
\hline & & Freq. & $\%$ & Freq. & $\%$ \\
\hline \multirow{2}{*}{ Norte } & PA & 3 & 1,43 & 14 & 1,15 \\
\hline & AM & 1 & 0,47 & 3 & 0,24 \\
\hline \multirow{7}{*}{ Nordeste } & $\mathrm{RN}$ & 5 & 2,39 & 13 & 1,07 \\
\hline & $\mathrm{CE}$ & 13 & 6,22 & 55 & 4,54 \\
\hline & PE & 7 & 3,35 & 34 & 2,81 \\
\hline & $\mathrm{BA}$ & 3 & 1,44 & 14 & 1,15 \\
\hline & $\mathrm{PI}$ & 1 & 0,47 & 2 & 0,16 \\
\hline & $\mathrm{AL}$ & 1 & 0,47 & 3 & 0,24 \\
\hline & MA & 1 & 0,47 & 6 & 0,48 \\
\hline \multirow{3}{*}{ Centro-Oeste } & GO & 7 & 3,29 & 31 & 2,56 \\
\hline & MS & 1 & 0,47 & 4 & 0,33 \\
\hline & DF & 7 & 3,29 & 26 & 2,15 \\
\hline \multirow{4}{*}{ Sudeste } & RJ & 19 & 9,09 & 189 & 15,62 \\
\hline & SP & 82 & 39,23 & 557 & 46,03 \\
\hline & MG & 21 & 10,04 & 85 & 7,02 \\
\hline & ES & 3 & 1,43 & 16 & 1,32 \\
\hline \multirow{3}{*}{ Sul } & PR & 12 & 5,74 & 55 & 4,55 \\
\hline & SC & 4 & 1,91 & 18 & 1,49 \\
\hline & RS & 18 & 8,61 & 85 & 7,02 \\
\hline Total & & 209 & 100,0 & 1.210 & 100,0 \\
\hline
\end{tabular}

Fonte: Comissão Nacional de Residência Médica 
Tabela 2. Distribuição das vagas, população, casos novos e óbitos por câncer, segundo região, no Brasil, em 2010

\begin{tabular}{|c|c|c|c|c|c|c|c|}
\hline Parâmetros & Região & Norte & Nordeste & $\begin{array}{l}\text { Centro- } \\
\text {-Oeste }\end{array}$ & Sudeste & Sul & Total \\
\hline \multirow{2}{*}{$\begin{array}{l}\text { Vagas } \\
\text { ofertadas }\end{array}$} & Freq. & 17 & 125 & 57 & 847 & 165 & 1211 \\
\hline & $\%$ & 1,40 & 10,32 & 4,70 & 69,94 & 13,62 & 100,0 \\
\hline \multirow{2}{*}{$\begin{array}{l}\text { População da } \\
\text { região }\end{array}$} & Freq. & 15.360 .000 & 53.591 .000 & 13.895 .000 & 80.916 .000 & 27.719 .000 & 191.481 .000 \\
\hline & $\%$ & 8,02 & 27,9 & 7,25 & 42,26 & 14,48 & 100 \\
\hline \multirow{2}{*}{ Casos novos } & Freq. & 19.120 & 89.350 & 30.340 & 247.980 & 102.480 & 489.270 \\
\hline & $\%$ & 3,9 & 18,2 & 6,2 & 50,68 & 20,9 & 100 \\
\hline \multirow{2}{*}{ Óbitos } & Freq. & 1.927 & 8.249 & 3.252 & 23.382 & 10.127 & 46.937 \\
\hline & $\%$ & 4,10 & 17,57 & 6,92 & 49,82 & 21,58 & 100 \\
\hline $\begin{array}{l}\text { Taxa de } \\
\text { incidência }\end{array}$ & Taxa * & 117,73 & 165,75 & 213,10 & 295,94 & 357,53 & 243,77 \\
\hline
\end{tabular}

* por 100.000 para ambos os sexos

Fonte: Ministério da Saúde, INCA, Comissão Nacional de Residência Médica

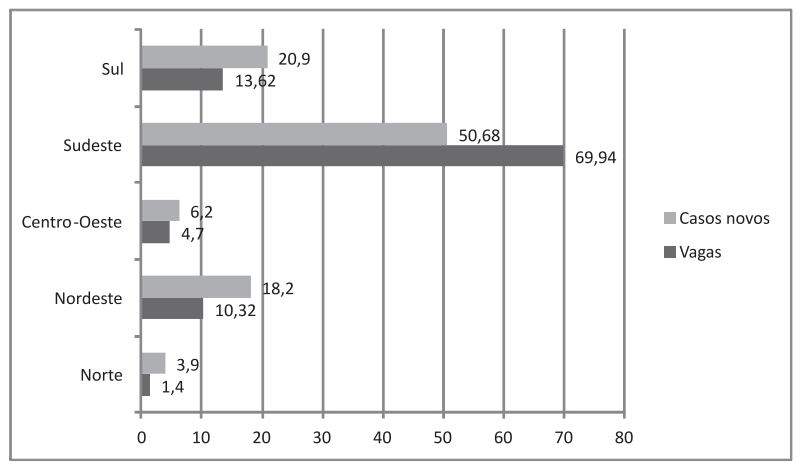

Figura 1. Distribuição das vagas de residência em oncologia (qualquer um dos programas) e do número estimado de casos novos de câncer, em percentagem, segundo região, no Brasil em 2010 Fonte: Comissão Nacional de Residência Médica

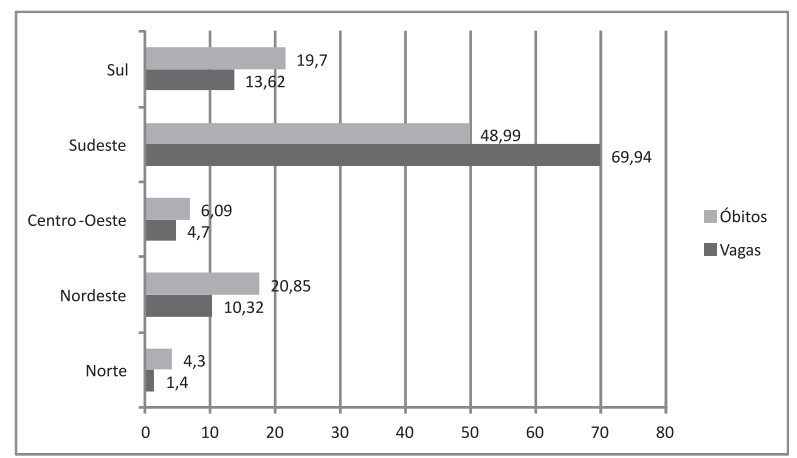

Figura 2. Distribuição das vagas de residência em cancerologia (qualquer um dos programas) e do número de óbitos por câncer, em percentagem, segundo região, no Brasil, em 2010

Fonte: Comissăo Nacional de Residência Médica

Estabeleceu-se um paralelo entre o número de vagas ofertadas nas áreas de cancerologia: clínica, pediátrica, cirúrgica, e radioterapia nos anos de 2003 e $2010 . \mathrm{Na}$ região Norte, entre 2003 e 2010, surgiram 12 vagas de residência médica nessas áreas. No Sul, o número de vagas quase dobrou, saindo de 50 vagas em 2003 para
98 vagas em 2010. O Centro-Oeste que, em 2003, possuía 20 vagas, foi a região com o menor incremento, chegando a 29 vagas em 2010. A regiāo Nordeste, antes detentora de 26 vagas em 2003, mais que duplicou seu número de vagas, chegando a $61 \mathrm{em} \mathrm{2010.} \mathrm{O} \mathrm{Sudeste}$ em 2003 detinha 265 vagas e em 2010 chegou a 489 vagas, contabilizando um incremento de, portanto, 84,5\%. Considerando-se apenas as áreas de cancerologia, oncologia clínica, oncologia pediátrica, oncologia cirúrgica e radioterapia, foi visto que, em 2003, em todo o Brasil, havia 361 vagas e que, em 2010, atingiu-se 689 vagas.

\section{DISCUSSÃO}

Os programas de residência médica experimentaram um grande crescimento, a partir da criação da CNRM, em 1977. Em 1982, o número de especialistas registrados nos arquivos da CNRM foi de 448; por outro lado, 10 anos depois a entidade credenciou 1.990 egressos, revelando um aumento de $344 \% 6$.

Comparando-se apenas as seguintes áreas: cancerologia, oncologia clínica, oncologia pediátrica, oncologia cirúrgica e radioterapia; percebeu-se que, entre 2003 e 2010, houve um aumento de $90,9 \%$ no número de vagas oferecidas nestas áreas.

Um importante desafio para os gestores de saúde é o de garantir a formaçáo e o emprego de especialistas em seu local de origem ${ }^{11}$. Mais incentivos deveriam ser criados em prol da retenção destes em suas cidades ou estados natais. Entretanto, não é isso o que se observa nas estatísticas deste trabalho.

Em 2010, existiam no País 209 programas de residência na área de cancerologia, contabilizando 1.211 vagas. A distribuição destes é bastante desequilibrada, percebendo-se uma concentração de $76,1 \%$ dos programas nas regiōes SulSudeste. Isso contribui ainda mais para aumentar a tradicional "migração" para os Estados do Sudeste, especialmente 
de profissionais da regiáo Nordeste, que vão em busca tanto dos melhores programas como também de maiores oportunidades de vaga. Além disso, uma vez residentes, parte desses migrantes acaba se instalando no Sudeste após sua residência médica. Confirma-se esse fato, quando se analisa a distribuição dos 1.457 cancerologistas do Brasil, em 2011, onde 36 atuavam na região Norte; 280, no Nordeste; 698, no Sudeste; 292, no Sul; e 151, no Centro-Oeste ${ }^{12}$.

Nos Estados Unidos, os salários médicos são avaliados de acordo com a hora de trabalho. Nesse país, nota-se uma grande valorização dos médicos oncologistas, onde ganham um dos maiores salários, com destaque para os radioterapeutas (radioterapia é uma das especialidades mais procuradas pelos recém-formados ${ }^{13}$, cuja hora de trabalho gira em torno de 126 dólares, ficando à frente, inclusive, dos cirurgiôes plásticos, que recebem 113 dólares por hora). Tal fato pode ser um dos contribuintes para fazer dessa naçáo uma das mais avançadas em recursos humanos na área de oncologia ${ }^{14}$.

Os CACON são a referência estabelecida para o tratamento do câncer. Para conseguir o credenciamento, tiveram que demonstrar infraestrutura, competência profissional e de processos. Em 2010, existiam 192 CACON, sendo somente $22,4 \%$ (43) destes ligados a programas de residência médica em oncologia. Isso indica uma persistência daquilo encontrado em 2003, quando se contabilizavam 182 CACON com $38(20,9 \%)$ deles ligados à residência médica. Esse pode ser um indicativo da escassez de recursos humanos capacitados para formação, da falta de tradição e/ou interesse na formação de recursos humanos nessa área ou, inclusive da falta de consciência das autoridades, em relação à necessidade de profissionais nessa área, ou até do entendimento do câncer, como um problema de saúde pública. Tais instituições perdem uma oportunidade impar, já que o fato de sediar uma residência estimula a atualizaçáo constante dos profissionais envolvidos na docência, criando um ambiente aberto à curiosidade científica e à renovação técnica.

As entidades filantrópicas congregadas na Associação Brasileira de Instituiçóes Filantrópicas de Combate ao Câncer (ABIFC) têm um papel histórico no combate ao câncer no Brasil. Em 2003, contabilizando-se somente as áreas de oncologia clínica, oncologia cirúrgica e radioterapia, tais instituiçôes abrigavam cerca de 30\% desses programas de residência médica no País. Em 2010, adicionando-se também ao cômputo de programas de residência médica em oncologia as áreas de cirurgia de cabeça e pescoço, mastologia e patologia; elas passaram a assumir $18 \%$ desses programas. Entretanto, apesar da queda percentual, houve um aumento importante no número de instituiçôes filantrópicas ligadas à oncologia, saindo de apenas cinco em 2003 para 19 em 2010.

Deve ser destacado, aqui, que a qualidade das informaçóes de base, sobre as quais são elaboradas as estimativas, pode estar contribuindo para a redução dos desequilíbrios na comparação com casos novos e óbitos esperados.

Existem critérios bastante claros para o credenciamento de um programa de residência pela CNRM, mas o que não está evidente é se existe um planejamento, em nível nacional, que leve em consideração as necessidades da população e que estabeleça programas de formação que respondam a essas necessidades, tal como ocorre em alguns países europeus, servindo de exemplo a Espanha, a Holanda e o Reino Unido.

A importância das neoplasias malignas vem crescendo no Brasil, na medida em que se observa um aumento da importância dos grupos expostos a doenças crônico-degenerativas ${ }^{15,16}$. Frente à expansão do câncer no País, cabe uma reflexáo acerca da necessidade de formação de especialistas que possam responder a essa demanda crescente.

\section{CONCLUSÃO}

As instituições com programas e vagas de residência médica em cancerologia e de CACON concentram-se no Sudeste, principalmente no Estado de São Paulo. A regiāo Sudeste é a única do País que possui um maior valor percentual de programas e vagas de residência médica na área da cancerologia em relação ao valor percentual que detém da populaçáo brasileira, do total de óbitos por câncer e da incidência de câncer.

As instituiçôes federais abrigam a maior parte dos programas de residência médica ligados à oncologia. Entre 2003 e 2010, notou-se que o desequilíbrio na distribuição de programas e da oferta de vagas persiste.

\section{CONTRIBUIÇÕES}

Todos os autores contribuíram na concepção e planejamento do estudo; na obtenção, análise e interpretação dos dados; e na redação e revisão crítica.

\section{Declaração de Conflito de Interesses: Nada a Declarar.}

\section{REFERÊNCIAS}

1. Ministério da Educação (Brasil). Residências em saúde. [Internet] 2012 [acesso 2012 abr 18]. Disponível em: http://portal.mec.gov.br/index.php?option=com_conte nt\&view=article\&id=12263\&Itemid $=506$

2. Machado JLM, Caldas Junior AL, Bortoncello NMF. Uma nova iniciativa na formação dos profissionais de saúde. Interface: comunicação, saúde, educação. 1997; 1(1):147-156.

3. Feuerwerker LCM. Mudanças na educação médica e residência médica. São Paulo: Hucitec; 1998. 190p. 
4. Hospital AC Camargo. Residência médica e aprimoramento profissional. [Internet]. São Paulo: AC Camargo; 2012. [acesso 2012 jun 20]. Disponível em: http://www.accamargo.org.br/residencia-medica/..

5. Silva MGC, Arregi MMU. Residência médica na área de cancerologia no Brasil: distribuição dos programas e da oferta de vagas por região em 2003. Revista bras de cancerol. 2005; 51(1):5-13.

6. Comissão Nacional de Residência Médica. Sistema CNRM: instituiçôes x programas x vagas. [Internet] 2012 [acesso 2012 abr 26]. Disponível em: http://mecsrv04. mec.gov.br/sesu/SIST_CNRM/APPS/cons_res_inst.asp

7. Instituto Nacional de Câncer (Brasil). Registro hospitalar de câncer. [Internet]. Rio de Janeiro: INCA; 2010. [acesso 2012 mai 10]. Disponível em: http://www.inca.gov.br/ conteudo_view.asp?id=351

8. Ministério da Saúde (Brasil). Indicadores e dados básicos - Brasil 2010: indicadores e dados básicos para a saúde. Rio de Janeiro: MS; 2010. 12p.

9. Departamento de Informática do SUS (DATASUS). Sistema de informação de mortalidade. [Internet] 2012. [acesso 2012 jun 18]. Disponível em: http://www2. datasus.gov.br/DATASUS/index.php?area=040701
10. Instituto Nacional de Câncer (Brasil). Estimativa 2010: incidência de câncer no Brasil. Rio de Janeiro: INCA; 2009.

11. Organización Panamericana de la Salud. Residencias médicas en América Latina. Washington; 2011. (La Renovación de la Atención Primaria de Salud en las Américas, 5). 127p

12. Scheffer M. Atlas da demografia médica no Brasil: unidades da federação. In: Scheffer M, coordenador. Demografia médica no Brasil. São Paulo: Conselho Federal de Medicina; 2011. p. 87-115.

13. Green M, Jones P, Thomas Jr JX. Selection criteria for residency: results of a National Program Directors Survey. Acad Med, 2009 Mar; 84(3):362-7.

14. Larson NF. Significant discrepancies in physician hourly wages exist across specialties. Arch Intern Med. 2010; 1728-1734, 1742-1744.

15. Carmo EH, Barreto ML, Silva Junior JB. Mudanças nos padrões de morbimortalidade da população brasileira: os desafios para um novo século. Epidemiol e Serv de Saúde. 2003; 12(2):63-75.

16. Schwartsmann G, Martelette M. Câncer. In: Duncan BB, Schmidt MI, Giugliani ERJ. Medicina ambulatorial: condutas clínicas em atenção primária. 3. ed. Porto Alegre: Artes Médicas; 2004. p.837-854. 


\section{Abstract}

Introduction: Medical residency is a post-graduation teaching modality, designed to doctors as specialized courses, characterized by in-service training, operating under the responsibility of health institutions, whether academic or not, under the guidance of highly qualified medical professionals. Objectives: To examine the provision of programs and vacancies of residency in Oncology, in Brazil, in 2010, by comparing it with estimated incidence and deaths from cancer, by regions; in addition, to compare the distribution of these programs, according to administrative dependence and to assess the distribution of High Complexity Centers in Oncology. Method: This was a descriptive and quantitative cross-sectional study. Data were arranged in sheets and correlated through statistical analysis. When indicated, a statistical test was done by Spearman correlation. Results: The Southeast gathers $59.8 \%$ of the programs; the South is home to $16.3 \%$, the Midwest, $7.18 \%$, the Northeast, $14.8 \%$, and the North, $1.91 \%$. Considering the areas of oncology, clinical oncology, pediatric oncology, surgical oncology and radiotherapy; it was seen that, between 2003 and 2010, there was an increase in vacancies of 90.86\%. Conclusion: The Southeast region has the most vacancies, programs and High Complexity Centers in Oncology. It's the only place that has a higher percentage of programs and vacancies in relation to the percentage of the Brazilian population each region has, and the total number of cancer incidence and deaths.

Key words: Medical Oncology-Education; Internship and Residency; Professional Practice; Inservice Training

\section{Resumen}

Introducción: La residencia médica es una modalidad de enseñanza de post-graduación, destinada a los médicos en forma de cursos de especialización, caracterizada por las prácticas en el servicio, trabajando bajo la responsabilidad de las instituciones de salud, académicas o no, bajo la orientación de profesionales médicos altamente cualificados. Objetivos: Analizar la oferta de programas y plazas de residencia médica en oncología en Brasil en 2010, comparada con la población estimada, incidencia, y óbitos por cáncer, por macro regiones, además de comparar la distribución de estos programas, según la dependencia administrativa y evaluar la distribución de los Centros de alta Complejidad en Oncología. Método: Se trata de un estudio descriptivo y cuantitativo del tipo transversal. Los datos obtenidos han sido dispuestos en plantillas y correlacionados a través del análisis estadístico. Cuando indicado, se hizo la prueba estadística por la correlación de Spearman. Resultados: La región Sudeste reúne el 59,8\% de los programas, la Sur detiene el 16,3\%, el Centro Oeste, 7,18\%, el Nordeste 14,8\% y la región Norte, 1,91\%. Teniendo en cuenta áreas de oncología, oncología clínica, oncología pediátrica, oncología quirúrgica y radioterapia, se notó que, entre 2003 y 2010 hubo un incremento de las plazas del 90,86\%. Conclusión: La región Sudeste detiene la mayoría de los programas, plazas y Centros de Alta Complejidad en Oncología. Es la única del País que posee el más alto porcentual de programas y plazas en relación al valor porcentual que detiene la población brasileña del total de óbitos por cáncer y de la incidencia del cáncer.

Palabras clave: Oncología Médica-Educación; Internado y Residencia; Práctica Profesional; Capacitación em Servicio 